\title{
Boundary knot method for heat conduction in nonlinear functionally graded material
}

\author{
Zhuo-Jia Fu ${ }^{\mathrm{a}, \mathrm{b}}$, Wen Chen ${ }^{\mathrm{a}, *}$, Qing-Hua Qin ${ }^{\mathrm{b}}$ \\ ${ }^{a}$ Center for Numerical Simulation Software in Engineering and Sciences, Department of Engineering Mechanics, Hohai University, Nanjing, Jiangsu, PR China \\ ${ }^{\mathrm{b}}$ School of Engineering, Building 32, Australian National University, Canberra ACT 0200, Australia
}

\section{A R T I C L E I N F O}

\section{Article history:}

Received 13 August 2010

Accepted 11 December 2010

\section{Keywords:}

Boundary knot method

Kirchhoff transformation

Nonlinear functionally graded material

Heat conduction

Meshless

\begin{abstract}
A B S T R A C T
This paper firstly derives the nonsingular general solution of heat conduction in nonlinear functionally graded materials (FGMs), and then presents boundary knot method (BKM) in conjunction with Kirchhoff transformation and various variable transformations in the solution of nonlinear FGM problems. The proposed BKM is mathematically simple, easy-to-program, meshless, high accurate and integration-free, and avoids the controversial fictitious boundary in the method of fundamental solution (MFS). Numerical experiments demonstrate the efficiency and accuracy of the present scheme in the solution of heat conduction in two different types of nonlinear FGMs.
\end{abstract}

(c) 2010 Elsevier Ltd. All rights reserved.

\section{Introduction}

Functionally graded materials (FGMs) are a new generation of composite materials whose microstructure varies from one material to another with a specific gradient. In particular, "a smooth transition region between a pure ceramic and pure metal would result in a material that combines the desirable high temperature properties and thermal resistance of a ceramic, with the fracture toughness of a metal" [1]. In virtue of their excellent behaviors, FGMs have become more and more popular in material engineering and have featured in a wide range of engineering applications (e.g., thermal barrier materials [2], optical materials [3], electronic materials [4] and ever biomaterials [5])

During the past decades extensive studies have been carried out on developing numerical methods for analyzing the thermal behavior of FGMs, for example, the finite element method (FEM) [6], the boundary element method (BEM) [7,8], the meshless local boundary integral equation method (LBIE) [9], the meshless local PetrovGalerkin method (MLPG) [10-13] and the method of fundamental solution (MFS) [14-16]. However, the conventional FEM is inefficient for handling materials whose physical property varies continuously; BEM needs to treat the singular or hyper-singular integrals $[17,18]$, which is mathematically complex and requires additional computing costs. It is worth noting that, with the exception of mesh-based FEM and BEM, the other above-mentioned methods are classified to the meshless method. Among these meshless methods, LBIE and

\footnotetext{
* Corresponding author.

E-mail address: chenwen@hhu.edu.cn (W. Chen).
}

MLPG belong to the category of weak-formulation, and MFS belongs to the category of strong-formulation.

This study focuses on strong-formulation meshless methods due to their inherent merits on easy-to-program and integrationfree. The MFS distributes the boundary knots on fictitious boundary [19] outside the physical domain to avoid the singularities of fundamental solutions, and selecting the appropriate fictitious boundary plays a vital role for the accuracy and reliability of the MFS solution, however, it is still arbitrary and tricky task, largely based on experiences.

Later, Chen and Tanaka [20] develops an improved method, boundary knot method (BKM), which used the nonsingular general solution instead of the singular fundamental solution and thus circumvents the controversial artificial boundary in the MFS. This study first derives the nonsingular general solution of heat conduction in FGM, and then applies the BKM in conjunction with the Kirchhoff transformation to heat transfer problems with nonlinear thermal conductivity. A brief outline of the paper is as follows: Section 2 describes the BKM coupled with Kirchhoff transformation for heat conduction in nonlinear FGM, followed by Section 3 to present and discuss the numerical efficiency and accuracy of the proposed approach in two typical examples. Finally some conclusions are summarized in Section 4.

\section{Boundary knot method for nonlinear functionally graded material}

Consider a heat conduction problem in an anisotropic heterogeneous nonlinear FGM, occupying a 2D arbitrary shaped region 
$\Omega \subset \mathfrak{R}^{2}$ bounded by its boundary $\Gamma$, and in the absence of heat sources. Its governing differential equation is stated as

$\sum_{i, j=1}^{2} \frac{\partial}{\partial x_{i}}\left(K_{i j}(x, T) \frac{\partial T(x)}{\partial x_{j}}\right)=0, \quad x \in \Omega$

with the following boundary conditions.

Dirichlet/essential condition:

$T(x)=\bar{T}, \quad x \in \Gamma_{D}$

Neumann/natural condition:

$q(x)=-\sum_{i, j=1}^{2} K_{i j} \frac{\partial T(x)}{\partial x_{j}} n_{i}(x)=\bar{q}, \quad x \in \Gamma_{N}$

Robin/convective condition:

$q(x)=h_{e}\left(T(x)-T_{\infty}\right), \quad x \in \Gamma_{R}$

where $T$ is the temperature, $\Gamma=\Gamma_{D}+\Gamma_{N}+\Gamma_{R}$ and $K=$ $\left\{K_{i j}(x, T)\right\}_{1 \leq i, j \leq 2}$ denotes the thermal conductivity matrix which satisfies the symmetry $\left(K_{12}=K_{21}\right)$ and positive-definite $\left(\Delta_{K}=\operatorname{det}(K)=K_{11} K_{22}-K_{12}^{2}>0\right)$ conditions. $\left\{n_{i}\right\}$ the outward unit normal vector at boundary $x \in \partial \Omega, h_{e}$ the heat conduction coefficient and $T_{\infty}$ the environmental temperature.

In this study, we assume the heat conductor is an exponentially functionally graded material such that its thermal conductivity can be expressed by

$K_{i j}(x, T)=a(T) \bar{K}_{i j} e^{\sum_{i=1}^{2} 2 \beta_{i} x_{i}}, \quad x=\left(x_{1}, x_{2}\right) \in \Omega$

in which $a(T)>0, \bar{K}=\left\{\bar{K}_{i j}\right\}_{1<i, j<2}$ is a symmetric positive definite matrix, and the values are all real constants. $\beta_{1}$ and $\beta_{2}$ denote constants of material property characteristics.

By employing the Kirchhoff transformation

$\phi(T)=\int a(T) d T$

Eqs. (1) and (2) can be reduced as the following form:

$\left(\sum_{i, j=1}^{2}\left(\bar{K}_{i j} \frac{\partial^{2} \Phi_{T}(x)}{\partial x_{i} \partial x_{j}}+2 \beta_{i} \bar{K}_{i j} \frac{\partial \Phi_{T}(x)}{\partial x_{j}}\right)\right) e^{\sum_{i=1}^{2} 2 \beta_{i} x_{i}}=0, \quad x \in \Omega$

$\Phi_{T}(x)=\phi(\bar{T}), \quad x \in \Gamma_{D}$

$q(x)=-\sum_{i, j=1}^{2} K_{i j} \frac{\partial T(x)}{\partial x_{j}} n_{i}(x)=-e^{\sum_{i=1}^{2} 2 \beta_{i} x_{i}} \sum_{i, j=1}^{2} \bar{K}_{i j} \frac{\partial \Phi_{T}(x)}{\partial x_{j}} n_{i}(x)=\bar{q}, x \in \Gamma_{N}$

$q(x)=h_{e}\left(\Phi_{T}(x)-\varphi\left(T_{\infty}\right)\right), \quad x \in \Gamma_{R}$

where $\Phi_{T}(x)=\varphi(T(x))$ and the inverse Kirchhoff transformation

$T(x)=\varphi^{-1}\left(\Phi_{T}(x)\right)$

And then we derive the nonsingular general solution of Eq. (5) by two-step variable transformations:

Step 1: To simplify the expression of Eqs. (5), let $\Phi_{T}=$ $\Psi e^{-\sum_{i=1}^{2} \beta_{i}\left(x_{i}+s_{i}\right)}$. Eq. (5) can then be rewritten as follows:

$\left(\sum_{i, j=1}^{2} \bar{K}_{i j} \frac{\partial \Psi(x)}{\partial x_{i} \partial x_{j}}-\lambda^{2} \Psi(x)\right) e^{\sum_{i=1}^{2} \beta_{i}\left(x_{i}+s_{i}\right)}=0, \quad x \in \Omega$

where $\lambda=\sqrt{\sum_{i=1}^{2} \sum_{j=1}^{2} \beta_{i} \bar{K}_{i j} \beta_{j}}$. Since $e^{\sum_{i=1}^{2} \beta_{i}\left(x_{i}+s_{i}\right)}>0$. The Trefftz functions of Eq. (8) are equal to those of anisotropic modified Helmholtz equation.
Step 2: To transform the anisotropic Eq. (8) into isotropic one, we set

$$
\left(\begin{array}{l}
y_{1} \\
y_{2}
\end{array}\right)=\left(\begin{array}{ll}
1 / \overline{\bar{K}}_{11} & 0 \\
-\bar{K}_{12} / \sqrt{\bar{K}_{11} \Delta_{\bar{K}}} & \sqrt{\bar{K}_{11}} / \sqrt{\Delta_{\bar{K}}}
\end{array}\right)\left(\begin{array}{l}
x_{1} \\
x_{2}
\end{array}\right)
$$

where $\Delta_{\bar{K}}=\operatorname{det}(\bar{K})=\bar{K}_{11} \bar{K}_{22}-\bar{K}_{12}^{2}>0$.

It follows from Eq. (8) that

$\left(\sum_{i=1}^{2} \frac{\partial^{2} \Psi(y)}{\partial y_{i} \partial y_{i}}-\lambda^{2} \Psi(y)\right)=0, \quad y \in \Omega$

Therefore, Eq. (10) is the isotropic modified Helmholtz equation, the corresponding nonsingular solution can be found in [20]. Then the nonsingular solution of Eq. (8) can be obtained by using inverse transformation (9),

$u_{G}(x, s)=-\frac{1}{2 \pi \sqrt{\Delta_{\bar{K}}}} I_{0}(\lambda R)$

in which $R=\sqrt{\sum_{i=1}^{2} \sum_{j=1}^{2} r_{i} \bar{K}_{i j}^{-1} r_{j}}, r_{1}=x_{1}-s_{1}, r_{2}=x_{2}-s_{2}$, where$x$, sare collocation points and source points, respectively, and $I_{0}$ denotes the zero-order modified Bessel function of first kind.

Finally, by implementing the variable transformation $\Phi_{T}=\Psi e^{-\sum_{i=1}^{2} \beta_{i}\left(x_{i}+s_{i}\right)}$, the nonsingular solution of Eq. (5) is in the following form:

$u_{G}(x, s)=-\frac{I_{0}(\lambda R)}{2 \pi \sqrt{\Delta_{\bar{K}}}} e^{-\sum_{i=1}^{2} \beta_{i}\left(x_{i}+s_{i}\right)}$

It is worth noting that the source points are placed on the physical boundary by using the present nonsingular general solution $u_{G}$.

In the boundary knot method, the solution of Eqs. (5) and (6) is approximated by a linear combination of general solutions with the unknown expansion coefficients as shown below:

$\bar{\Phi}(x)=\sum_{i=1}^{N} \alpha_{i} u_{G}\left(x, s_{i}\right)$

where $\left\{\alpha_{i}\right\}$ are the unknown coefficients determined by boundary conditions. After $\Phi(x)$ is obtained, the temperature solution $T$ to Eqs. (1) and (2) can be obtained using Eq. (7).

The heat flux can then be given by

$q(x)=\sum_{i=1}^{N} \alpha_{i} Q\left(x, s_{i}\right)$

in which

$$
\begin{aligned}
& Q\left(x, s_{i}\right)=\sum_{i, j=1}^{2} \bar{K}_{i j} \frac{\partial u_{G}\left(x, s_{i}\right)}{\partial x_{j}} n_{i}(x) e^{\sum_{i=1}^{2} 2 \beta_{i} x_{i}} \\
& =\frac{e^{\sum_{i=1}^{2} 2 \beta_{i} r_{i}}}{2 \pi \sqrt{\Delta_{\bar{K}}}}\left(-\frac{\lambda}{R} I_{1}(\lambda R) \sum_{i=1}^{2} n_{i}(x) r_{i}+I_{0}(\lambda R) \sum_{i=1}^{2} \sum_{j=1}^{2} n_{i}(x) \bar{K}_{i j} \beta_{j}\right)
\end{aligned}
$$

where $I_{1}$ denotes the first-order modified Bessel function of first kind.

In view of the general solution satisfying the governing Eq. (5), a priori, the presented method only needs boundary discretization to satisfy boundary conditions

$A \alpha=b$

in which

$A=\left(\begin{array}{l}u_{G}\left(x_{j}, s_{i}\right) \\ Q\left(x_{j}, s_{i}\right) \\ Q\left(x_{j}, s_{i}\right)-h_{e} u\left(x_{j}, s_{i}\right)\end{array}\right)$ 
$\alpha=\left(\alpha_{1}, \alpha_{2}, \ldots, \alpha_{N}\right)^{T}$

$b=\left(\begin{array}{l}\varphi\left(\bar{T}_{j}\right) \\ \bar{q}_{j} \\ -h_{e} \varphi\left(\left(T_{\infty}\right)_{j}\right)\end{array}\right)$

where source points $s_{i} \in \partial \Omega, i=1,2, \ldots, N$, coinciding with the collocation points $x_{j} \in \partial \Omega, j=1,2, \ldots, M$. In other words, unlike the MFS, the BKM places the source points and collocation points on the same set of boundary knots, and $M=N$.

\section{Numerical results and discussions}

In this section, the efficiency, accuracy and convergence of the proposed BKM are assessed by considering two heat conduction problems in functionally graded materials (FGMs). The performance of the proposed method is compared with MFS solution and analytical solution. $\operatorname{Rerr}(w)$ and $\operatorname{Nerr}(w)$ defined below represent average relative errorand normalized error, respectively:

$\operatorname{Rerr}(w)=\sqrt{\frac{1}{N T} \sum_{i=1}^{N T}\left|\frac{w(i)-\bar{w}(i)}{\bar{w}(i)}\right|^{2}}$

$\operatorname{Nerr}(w)=\frac{|w(i)-\bar{w}(i)|}{\max _{1 \leq i \leq N T}|\bar{w}(i)|,}$

where $\bar{u}(i)$ and $u(i)$ are the analytical and numerical solutions at $x_{i}$, respectively, and NT denotes the total number of uniform test points in the interest domain. Unless otherwise specified, $N T$ is taken to be 100 in all following numerical cases.

Example 1. Consider the heat transfer in a nonlinear exponential heterogeneous FGM [16] whose coefficients of heat conduction are defined by Eq. (3) with $a(T)=e^{T}$. This example always occurs in high-temperature environments. Using Kirchhoff transformation, we can obtain $\Phi_{T}=e^{T}, T=\varphi^{-1}\left(\Phi_{T}\right)=\ln \left(\Phi_{T}\right)$.

Let us consider an orthotropic material in the square $\Omega=(-1,1) \times$ $(-1,1)$ in which $\bar{K}=\left(\begin{array}{ll}2 & 0 \\ 0 & 1\end{array}\right)$ and $\beta_{1}=0, \beta_{2}=1$. The analytical solution is

$T(x)=\ln \left(\sqrt{\frac{1-T x / T r}{2 T r}} \sinh (\operatorname{Tr}) e^{-T y}\right)$

$\Phi_{T}(x)=e^{T(x)}$

where $T x=x_{1} / \sqrt{2}-1, T y=x_{2}, T r=\sqrt{T x^{2}+T y^{2}}$.

Fig. 1(a) shows the condition number of interpolation matrix in Example 1 with respect to the number of boundary knots by using BKM and MFS with different fictitious boundary parameters. The condition number Cond in Fig. 1(a) is defined as the ratio of the largest and smallest singular value. It is observed that with increasing boundary points, the condition numbers of both the BKM and the MFS grow rapidly, which downplay these two methods. This illconditioned matrix problem is always found in the other collocation techniques, such as the Trefftz method [21] and the Kansa method [22]. There are several ways to handle this ill-conditioning problem, including the domain decomposition method [23], preconditioning technique based on approximate cardinal basis function, the fast multiple method [24] and regularization methods [25] such as the truncated singular value decomposition (TSVD).

This study will use the TSVD to mitigate the effect of bad conditioning in the BKM and MFS solutions, and the generalized cross-validation (GCV) function choice criterion is employed to estimate an appropriate regularization parameter of the TSVD. Our computations use the MATLAB SVD code developed by Hansen [25].
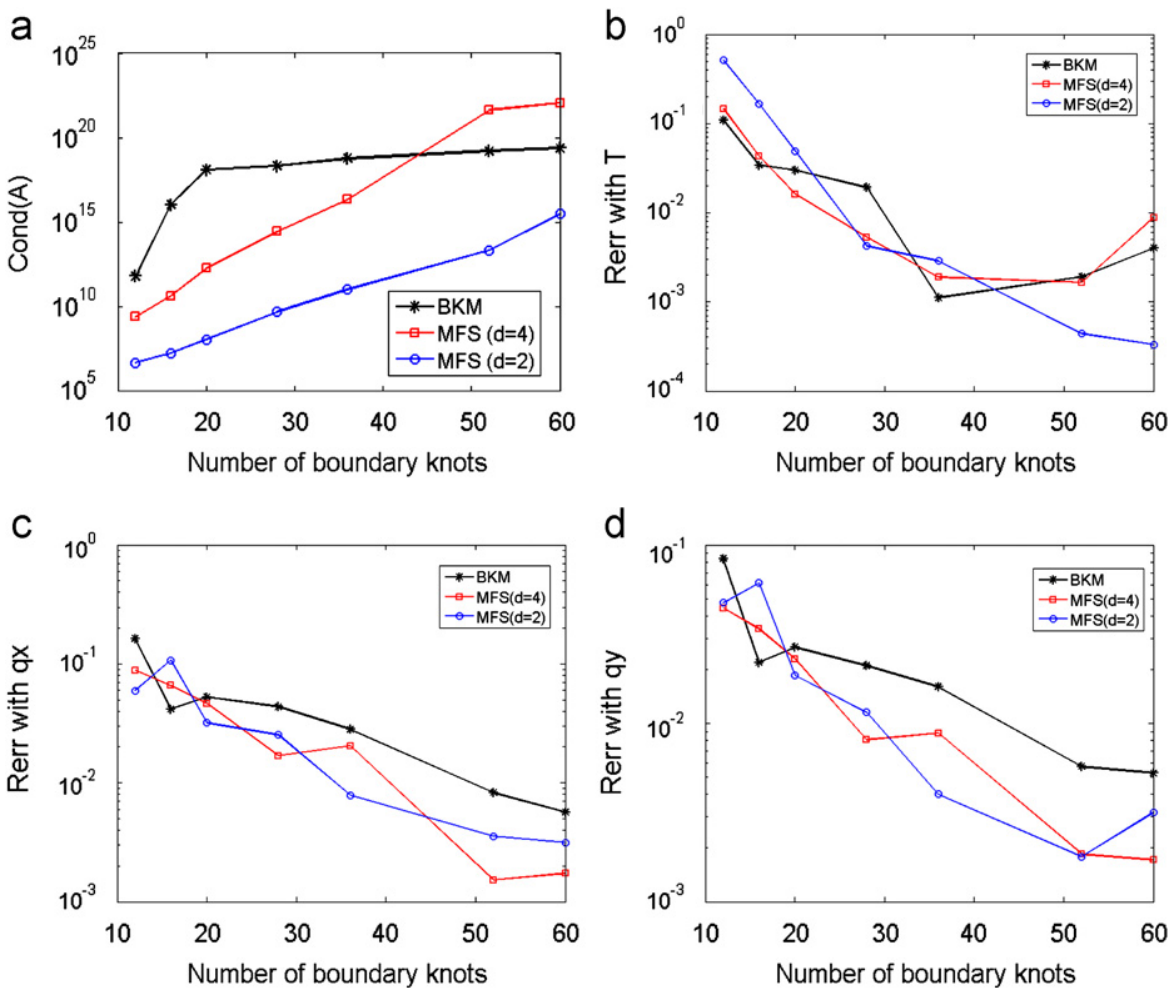

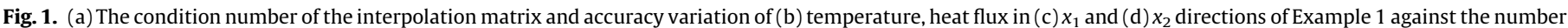
of boundary knots by BKM and MFS with different fictitious boundary parameters ( $d=2$ and 4 ). 


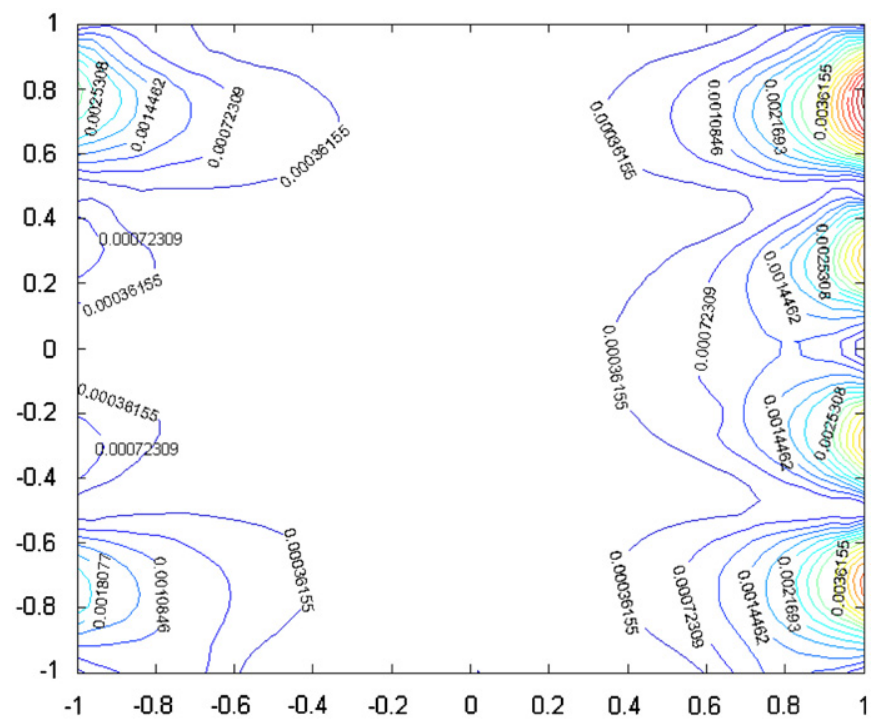

Fig. 2. Isolines of normalized errors of temperature by 20 boundary nodes BKM in Example 1.

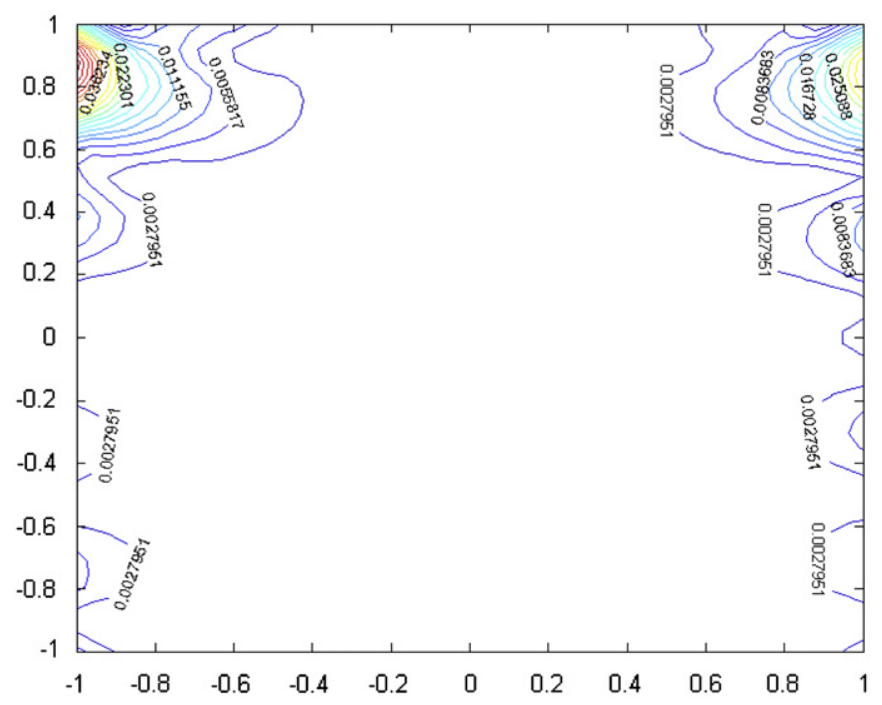

Fig. 3. Isolines of normalized errors of heat flux in the $x_{1}$ direction by 20 boundary nodes BKM in Example 1.

By implementing BKM and MFS coupled with the TSVD to solve the ill-conditioning matrix system, Fig. 1(b)-(d) shows the numerical accuracy variation of temperature, heat flux in the $x_{1}$ and $x_{2}$ directions, respectively, against the number of boundary collocation points $N$. As compared with MFS in Example 1, in general, the BKM has roughly similar degrees of accuracy compared with the MFS in heat flux fields. It can be found from Fig. 1 that the BKM yields more accurate solution than MFS with few knots, however, with further increasing knots, the BKM solution can not improve the accuracy better than MFS. This may result from that the BKM interpolation matrix becomes much worse than MFS.

Figs. 2-4 show the distribution of normalized errors of temperature and heat flux in the $x_{1}$ and $x_{2}$ directions, respectively, by using 20 boundary knots BKM in Example 1. It can be observed that the BKM results are in good agreement with the analytical solution. Nevertheless the BKM solution errors tend to become worse from the central to the boundary-adjacent regions, especially at boundary corners.

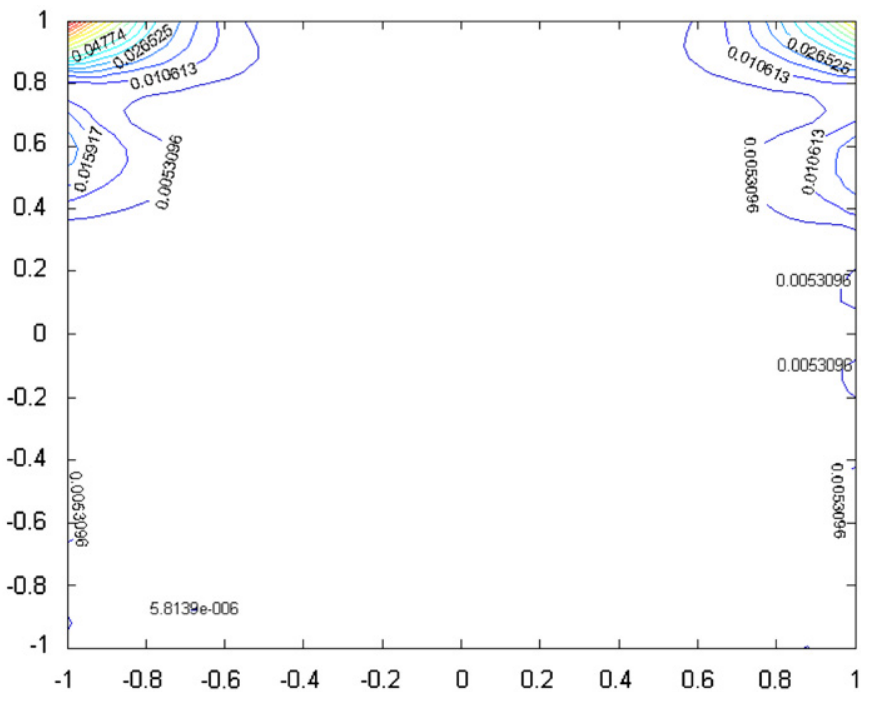

Fig. 4. Isolines of normalized errors of heat flux in the $x_{2}$ direction by 20 boundary nodes BKM in Example 1.

Example 2. This example considers another type of nonlinear exponential heterogeneous FGM in the same geometry $\Omega=(-1,1) \times(-1,1)$. In practice, the dependence of the thermal conductivity on the temperature may be chosen as linear, i.e., $a(T)=1+\mu T$, where $\mu$ is a constant. By using Kirchhoff transformation, we can obtain $\Phi_{T}=T+(\mu / 2) T^{2}, T=\varphi^{-1}\left(\Phi_{T}\right)=$ $-1+\sqrt{1+2 \mu \Phi_{T}} / \mu$.

The analytical solution in this example is

$$
\begin{aligned}
& T(x)=\frac{-1+\sqrt{1+2 \mu \Phi_{T}(x)}}{\mu} \\
& \Phi_{T}(x)=e^{\lambda(T x+T y) / \tau-\sum_{i=1}^{2} \beta_{i} x_{i}}
\end{aligned}
$$

in which

$\tau=\sqrt{\bar{K}_{11}\left(\frac{\sqrt{\Delta_{\bar{K}}}-\bar{K}_{12}}{\bar{K}_{11}}\right)^{2}+2 \bar{K}_{12}\left(\frac{\sqrt{\Delta_{\bar{K}}}-\bar{K}_{12}}{\bar{K}_{11}}\right)+\bar{K}_{22}}$

$T x=\frac{x_{1} \sqrt{\Delta_{\bar{K}}}}{\bar{K}_{11}}, T y=-\frac{x_{1} \bar{K}_{12}}{\bar{K}_{11}}+x_{2}$

where

$\bar{K}=\left(\begin{array}{ll}1 & 0.25 \\ 0.25 & 3\end{array}\right)$

and $\beta_{1}=0.1, \beta_{2}=0.8$, and $\mu=1 / 4$.

As in Example 1, Fig. 5(a) shows that the condition numbers of BKM and MFS interpolation matrices in Example 2 increase rapidly with the increasing boundary knots. Fig. 5(b)-(d) shows the convergent rate of temperature and heat flow in Example 2 by using BKM and MFS coupled with the TSVD. From these figures, it can be seen that the BKM has better performance with few interpolation knots than MFS. It is noted that the BKM solution accuracy improves evidently with modestly increasing boundary knots, but enhances slowly with a relatively large number of nodes characterized by visible oscillations, due to the severely ill-conditioned matrix.

On the other hand, the fictitious boundary in the MFS affects its numerical accuracy and stability in a remarkable way. It can be observed from the above figures that the MFS with a larger parameter $d(d=4)$, which characterizes the distance between 

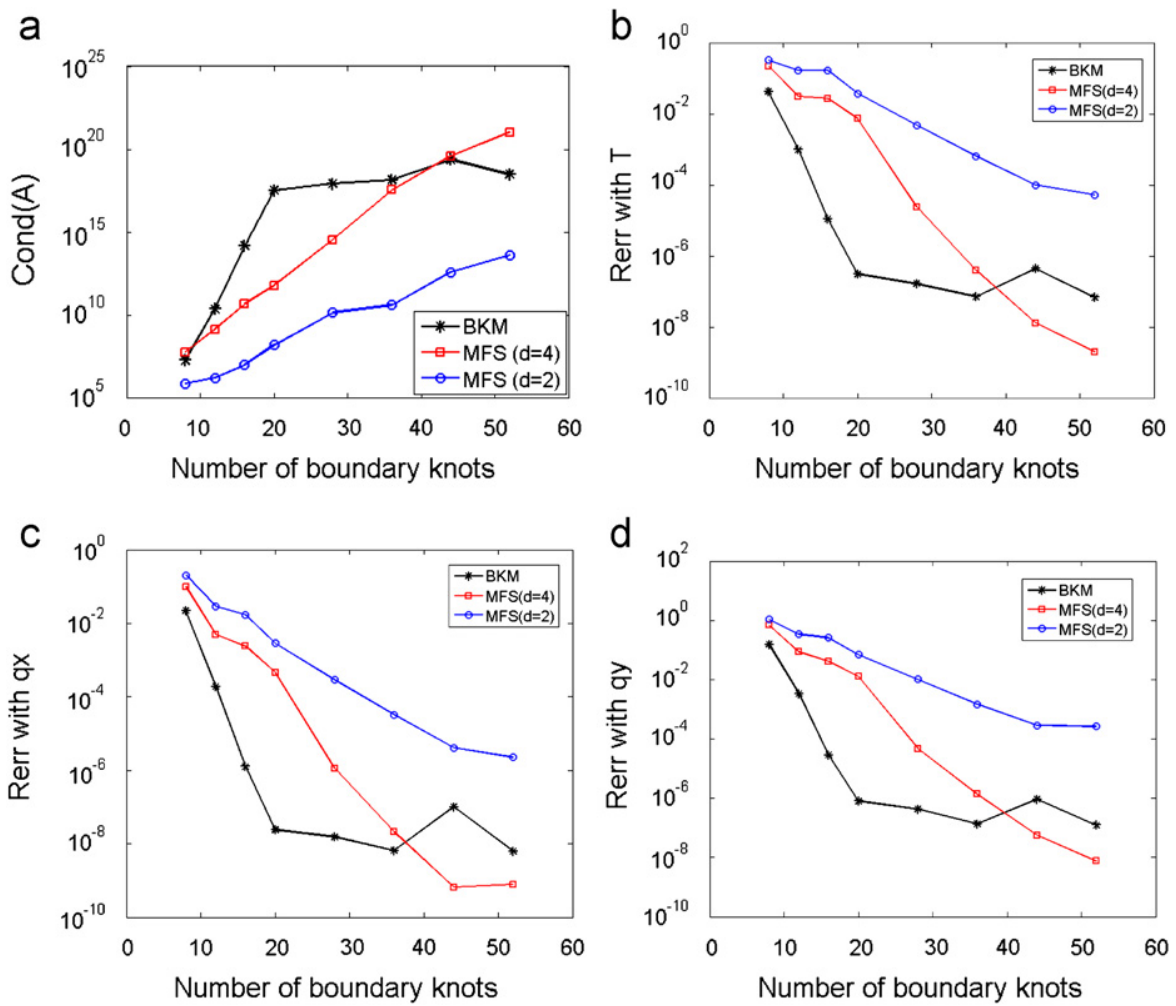

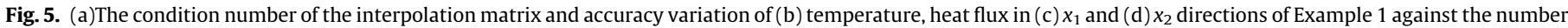
of boundary knots by BKM and MFS with different fictitious boundary parameters $(d=2$ and 4$)$.

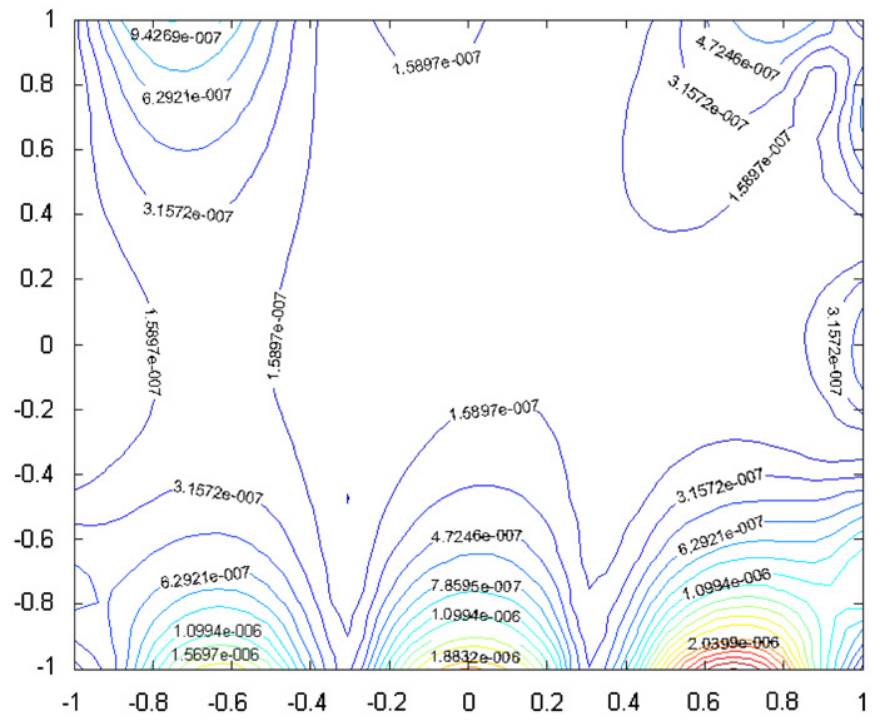

Fig. 6. Isolines of normalized errors of temperature by 16 boundary nodes BKM in Example 2.

the fictitious and real boundaries, can obtain more accurate solution than with small parameter $d=2$ in this example. However, in some cases the placement of the fictitious boundary far from the physical domain can lead to numerical instability or ever wrong solutions in the MFS [26]. In practical applications, the determination of the fictitious boundary is still quite tricky and often troublesome, especially in multi-connected and irregular domain problems. Therefore, the proposed method has the advantage over the MFS in that no fictitious boundary is required at all.

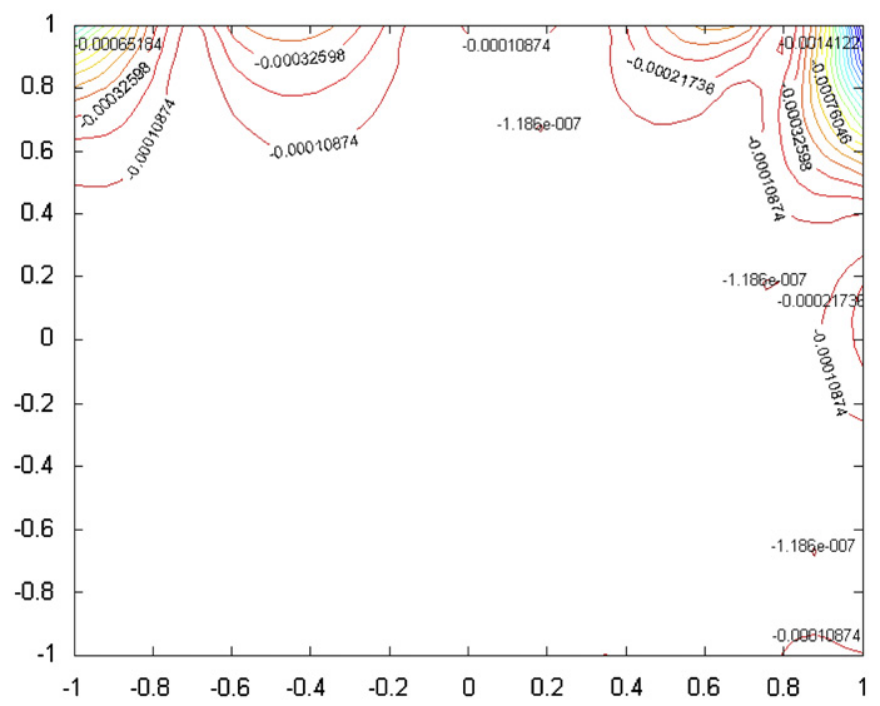

Fig. 7. Isolines of normalized errors of heat flux in the $x_{1}$ direction by 16 boundary nodes BKM in Example 2.

Figs. 6-8 represent the distribution of normalized errors of temperature and heat flux in the $x_{1}$ and $x_{2}$ directions, respectively, by using 16 boundary knots BKM in Example 2. It can be found that the proposed method provides very accurate approximations of the temperature and heat flux fields. As in Example 1, the errors at boundary-adjacent region are also worse than the central region. It is noted that this phenomena is always found in the other collocation techniques, such as the MFS, the Trefftz method and the Kansa method. 


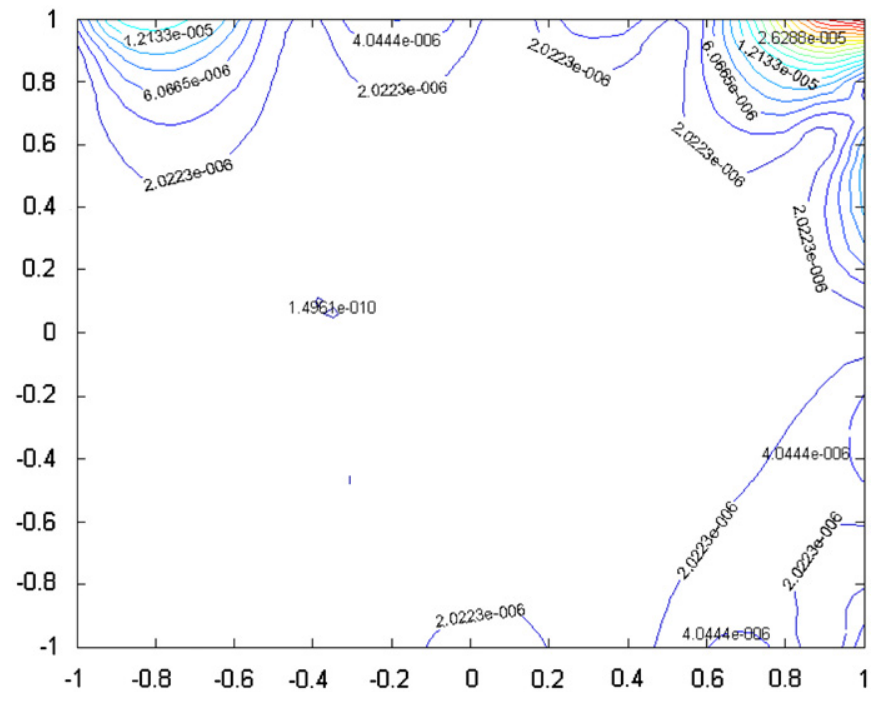

Fig. 8. Isolines of normalized errors of heat flux in the $x_{2}$ direction by 16 boundary nodes BKM in Example 2.

\section{Conclusions}

This paper presents the nonsingular general solution for two-dimensional heat conduction problems in exponential FGMs by way of the Kirchhoff transformation and coordinate transformations. The boundary knot method in conjunction with the truncated singular value decomposition is used for heat conduction analysis in nonlinear FGMs. Numerical demonstrations show that the proposed BKM is a competitive boundary collocation numerical method for the solution of heat conduction in nonlinear FGMs, which is mathematically simple, easy-to-program, meshless, high accurate and integration-free, and avoids the controversial fictitious boundary in the MFS. Future extension of the proposed method can be made to cases of three-dimensional composite materials [27] and transient heat transfer problems in FGMs $[10,28,29]$.

\section{Acknowledgements}

The work described in this paper was supported by National Basic Research Program of China (973 Project no. 2010CB832702), and the R\&D Special Fund for Public Welfare Industry (Hydrodynamics, Project no. 201101014) and Foundation for Open Project of the State Key Laboratory of Structural Analysis for Industrial Equipment (Project no. GZ0902). The first author would like to thank the Fundamental Research Funds for the Central Universities (Project no. 2009B03214) and the China Scholarship Council (CSC) for financial support.

\section{References}

[1] Gray LJ, Kaplan T, Richardson JD, Paulino GH. Green's functions and boundary integral analysis for exponentially graded materials: heat conduction. Journal of Applied Mechanics-Transactions of the ASME 2003;70(4):543-9.

[2] Erdogan F. Fracture mechanics of functionally graded materials. Composites Engineering 1995;5(7):753-70.

[3] Koike Y. High-bandwidth graded-index polymer optical fibre. Polymer 1991;32(10):1737-45.
[4] Tani J, Liu GRSH. Surface waves in functionally gradient piezoelectric plates. Tokyo, Japan: Japan Society of Mechanical Engineers; 1993.

[5] Pompe W, Worch H, Epple M, Friess W, Gelinsky M, Greil P, et al. Functionally graded materials for biomedical applications. Materials Science and Engineering A 2003;362(1-2):40-60.

[6] Kim J-H, Paulino GH. Isoparametric graded finite elements for nonhomogeneous isotropic and orthotropic materials. Journal of Applied Mechanics 2002;69(4):502-14.

[7] Sutradhar A, Paulino GH. The simple boundary element method for transient heat conduction in functionally graded materials. Computer Methods in Applied Mechanics and Engineering 2004;193(42-44):4511-39.

[8] Azis MI, Clements DL. Nonlinear transient heat conduction problems for a class of inhomogeneous anisotropic materials by BEM. Engineering Analysis with Boundary Elements 2008;32(12):1054-60.

[9] Sladek J, Sladek V, Zhang C. Transient heat conduction analysis in functionally graded materials by the meshless local boundary integral equation method. Computational Materials Science 2003;28(3-4):494-504.

[10] Sladek V, Sladek J, Tanaka M, Zhang C. Transient heat conduction in anisotropic and functionally graded media by local integral equations. Engineering Analysis with Boundary Elements 2005;29(11):1047-65.

[11] Sladek J, Sladek V, Hellmich C, Eberhardsteiner J. Heat conduction analysis of 3-D axisymmetric and anisotropic FGM bodies by meshless local PetrovGalerkin method. Computational Mechanics 2007;39(3):323-33.

[12] Sladek J, Sladek V, Atluri SN. Meshless local Petrov-Galerkin method for heat conduction problem in an anisotropic medium. Computer Modeling in Engineering and Sciences 2004;6:309-18.

[13] Sladek J, Sladek V, Tan CL, Atluri SN. Analysis of transient heat conduction in 3D anisotropic functionally graded solids by the MLPG. Computer Modeling in Engineering and Sciences 2008;32:161-74.

[14] Wang H, Qin QH, Kang YL. A meshless model for transient heat conduction in functionally graded materials. Computational Mechanics 2006;38(1):51-60.

[15] Wang H, Qin Q-H. Meshless approach for thermo-mechanical analysis of functionally graded materials. Engineering Analysis with Boundary Elements 2008;32(9):704-12.

[16] Marin L, Lesnic D. The method of fundamental solutions for nonlinear functionally graded materials. International Journal of Solids and Structures 2007;44(21):6878-90.

[17] Qin QH. Nonlinear-analysis of Reissner plates on an elastic-foundation by the BEM. International Journal of Solids and Structures 1993;30(22):3101-11.

[18] Brebbia CA, Telles JCF, Wrobel LC. Boundary element techniques: theory and applications in engineering/C.A. Brebbia, J.C.F. Telles, L.C. Wrobel. Berlin, New York: Springer-Verlag; 1984

[19] Fairweather G, Karageorghis A. The method of fundamental solutions for elliptic boundary value problems. Advances in Computational Mathematics 1998;9(1-2):69-95.

[20] Chen W, Tanaka M. A meshless, integration-free, and boundary-only RBF technique. Computers and Mathematics with Applications 2002;43(35):379-91.

[21] Zi-Cai L, Tzon-Tzer L, Hung-Tsai H, Alexander HDC. Trefftz, collocation, and other boundary methods-a comparison. Numerical Methods for Partial Differential Equations 2007;23(1):93-144.

[22] Kansa EJ. Multiquadrics - a scattered data approximation scheme with applications to computational fluid-dynamics - I. Surface approximations and partial derivative estimates. Computers and Mathematics with Applications 1990;19(8-9):127-45.

[23] Kansa EJ, Hon YC. Circumventing the ill-conditioning problem with multiquadric radial basis functions: applications to elliptic partial differential equations. Computers and Mathematics with Applications 2000;39(7-8): 123-37.

[24] Beatson RK, Cherrie JB, Mouat CT. Fast fitting of radial basis functions: methods based on preconditioned GMRES iteration. Advances in Computational Mathematics 1999;11(2):253-70.

[25] Hansen P. Regularization tools: a Matlab package for analysis and solution of discrete ill-posed problems. Numerical Algorithms 1994;6(1):1-35.

[26] Chen W, Fu ZJ, Wei X. Potential problems by singular boundary method satisfying moment condition. Computer Modeling in Engineering and Sciences 2009;54(1):65-85.

[27] Berger JR, Martin PA, Mantic V, Gray LJ. Fundamental solutions for steady-state heat transfer in an exponentially graded anisotropic material. Zeitschrift Fur Angewandte Mathematik und Physik 2005;56(2):293-303.

[28] Kuo HY, T.Y. Chen. Steady and transient Green's functions for anisotropic conduction in an exponentially graded solid. International Journal of Solids and Structures 2005;42(3-4):1111-28.

[29] Sutradhar A, Paulino GH, Gray LJ. Transient heat conduction in homogeneous and non-homogeneous materials by the Laplace transform Galerkin boundary element method. Engineering Analysis with Boundary Elements 2002;26(2):119-32. 